\title{
solo \\ Bases públicas de datos morfométricos en antropología biológica: nuevas prácticas, nuevos desafíos
}

RMA

Antropología Biológica

\author{
Paula N. González* y Marien Béguelin** \\ * Department of Cell Biology and Anatomy, University of Calgary. 3280 Hospital \\ Drive NW, Calgary, AB, Canadá T2N 4 Z6 e Instituto de Genética Veterinaria. \\ IGEVET-CONICET. La Plata, Argentina. \\ ** Instituto de Investigaciones en Paleobiología y Geología-Universidad Nacional
} de Río Negro

\begin{abstract}
Resumen
En los últimos años se observa una tendencia creciente hacia el desarrollo de prácticas que garanticen un acceso libre al conocimiento científico producido a través del aporte de fondos públicos. Este movimiento conocido como open access está ampliamente desarrollado en algunas disciplinas como la genética y la biología molecular, sin embargo en la antropología biológica su discusión es incipiente. El presente trabajo tiene por objetivo presentar algunas de las ventajas de esta política, tanto para los científicos como para la comunidad en general, y discutir un conjunto de aspectos prácticos que requieren ser considerados para la implementación del libre acceso a los datos (data sharing) en el seno de la antropología biológica. Particularmente, se discute acerca de la forma de obtención, almacenamiento, publicación y posterior uso de datos morfométricos en formato numérico, coordenadas 2 y 3D e imágenes digitales. Finalmente, se delinea una agenda de trabajo a fin de promover la discusión sobre el data sharing en nuestro pais.
\end{abstract}

Palabras Clave: acceso libre; conocimiento científico; datos antropométricos; imágenes digitales.

Morphometric data base in biological anthropology: new practices and challenges

\begin{abstract}
Over the last two decades there has been an increased interest in promoting the open access to scientific databases, predominantly those obtained in the context of the publicly funded science system. The main objective of this contribution is to review the advantages of this policy for the scientists as well as the general public. We point out some issues regarding to data collection, storage and publication, which still remain to be solved in order to implement the policy of data sharing within biological anthropology. Particularly, we focus on traditional and geometric morphometric data, and the new technologies for gathering digital images in 2D and 3D. We finally outline a set of points that need to be addressed in order to promote the discussion about data sharing in Argentina.
\end{abstract}

Keywords: open access; scientific knowledge; anthropometric data; data sharing; virtual collections.

"I have begun to think that no one ought to publish biometric results, without lodging a well-arranged and well-bound manuscript copy of his data in some place where it should be accessible" (Galton, Biometrika, n. 1, 1901)

Las palabras de Galton (1901) expresan el interés temprano de la ciencia por compartir libremente los datos (data sharing) producto de la investigación científica y con el avance reciente en las tecnologías de la comunicación este interés se ha revitalizado considerablemente. En los últimos años se han producido importantes cambios en las formas de difusión y publicación del conocimiento científico observándose una tendencia creciente hacia el desarrollo de prácticas que garanticen el acceso libre al conocimiento producido a través del aporte de fondos públicos, movimiento conocido como Open Access (Lossau 2008). En algunas disciplinas, como la genética y la biología molecular, la accesibilidad a datos normalizados a través de repositorios públicos resulta, actualmente, una práctica común y para tal fin se han desarrollado plataformas -tales como GenBank y treeBASE- que permiten almacenar y explorar datos relacionales. Por el contrario, otras áreas del conocimiento se encuentran aún en las etapas iniciales de la distribución libre de los datos, y solo recientemente algunas revistas han establecido como requisito para la publicación de los trabajos que los datos se encuentren archivados en repositorios públicos de acceso abierto y disponibles on line para la comunidad científica (Fairbairn 2010; Moore 
et al. 2010; Rausher et al. 2010; Rieseberg et al. 2010).

En este contexto la antropología biológica se encuentra aún lejos de contar con procedimientos estandarizados para la recolección, almacenamiento y publicación de los datos que garanticen su reutilización por el resto de los investigadores (Gilissen 2009; Kullmer 2008). En el marco de la reunión anual de la American Association of Physical Anthropology, los editores de dos de las revistas internacionales más importantes de la disciplina (Journal of Human Evolution y American Journal of Physical Anthropology) han abierto la discusión sobre las ventajas y desventajas de compartir datos, aunque aún no se ha alcanzado un consenso acerca de los procedimientos requeridos para implementar la creación de bases de datos antropológicos (Anton y Ruff 2011).

El objetivo del presente trabajo es discutir los alcances de la implementación de una política de acceso abierto a los datos, particularmente los datos morfométricos, en el seno de la Antropología Biológica. Cabe aclarar que si bien el trabajo se centra en un tipo particular de datos, la problemática abordada es de interés para conjuntos de datos más amplios. En primer lugar, se exponen los fundamentos del data sharing en el contexto del movimiento open access. A continuación, se examina el impacto que este movimiento tiene en las prácticas de recolección y almacenamiento de datos morfométricos. Finalmente, se evalúa el estado actual del libre acceso a los datos en nuestro país y se delinean las acciones necesarias a fin de extender estas prácticas al campo de la Antropología Biológica.

\section{Publicación de bases de datos en el contexto del libre acceso al conocimiento}

Los fundamentos del libre acceso a los datos generados por la comunidad científica pueden trazarse en el contexto más amplio del acceso abierto (Open Access) al conocimiento científico (Arzberger et al. 2004; Uhlir y Schröder 2007). Los debates actuales refieren principalmente al conocimiento académico producido a través del aporte de fondos públicos y parten de reconocer la creciente importancia del conocimiento para el desarrollo igualitario de los individuos y las sociedades. El término "conocimiento", incluye no sólo la publicación de los resultados sino también todos los datos en los que se sustenta la investigación. Estos principios han sido debatidos en foros de distintas organizaciones como la United Nations Educational, Scientific and Cultural Organization (UNESCO, 2008) y la Organisation for Economic Co-Operation and Development (OECD, 2007), entre otros.

Los beneficios de compartir datos (data sharing) son ampliamente reconocidos (Arzberger et al. 2004). Se ha señalado que el libre acceso a los mismos redundaría en una optimización de los fondos invertidos en el financiamiento de las investigaciones científicas al evitar la réplica de trabajos previamente realizados, incrementándose de esta manera los fondos destinados a trabajos originales. Asimismo, la práctica de compartir bases de datos presenta una serie de ventajas para la comunidad científica presente y futura, entre ellas la posibilidad de que otros investigadores puedan replicar los análisis y la preservación de información para futuros estudios que de otra forma se perdería (Whitlock 2011). Esto resulta en una mayor transparencia en el trabajo científico y minimiza la posibilidad de fraudes. Se argumenta también que desde el punto de vista del investigador que hace públicos sus datos, esta práctica conduciría a un aumento de los índices de citación con el concomitante beneficio de posicionarse mejor en las convocatorias para recibir financiamiento. Asimismo, incrementaría las propuestas de trabajo colaborativo y coautoría y permitiría incrementar la realización de trabajos de síntesis, dado que los conjuntos de datos disponibles serían más amplios y diversos (Rodrigo et al. 2013). Por último, desde la perspectiva de los denominados países periféricos esta práctica pondría a disposición de los investigadores datos obtenidos con tecnologías que generalmente se encuentran fuera de su alcance.

A pesar del acuerdo general respecto a las ventajas del data sharing, uno de los mayores debates gira actualmente en torno a cómo efectivizar estas prácticas. La infraestructura necesaria para mantener repositorios disciplinares requiere destinar fondos y recursos por parte de las propias agencias de financiación o las sociedades y revistas científicas. Con la excepción de algunas iniciativas como la del National Institute of Health de los Estados Unidos, que exige la publicación a través de Pubmed Central de los datos obtenidos por los proyectos que financia, existen muy pocos repositorios normalizados. En este sentido, consideramos que es fundamental que los organismos públicos como universidades y agencias gubernamentales de promoción de la investigación científica recuperen un rol central no solo en la producción del conocimiento, sino también en su difusión y en la obligación de poner a disposición pública los datos obtenidos con financiamiento público (Torres-Salinas 2010).

\section{Data sharing: el caso de los datos morfométricos en antropología}

Como se señaló anteriormente, uno de los principales objetivos de la creación de bancos de datos de acceso público es posibilitar integración de bases generadas por distintos investigadores, es decir la posibilidad de realizar meta-análisis. En este sentido, resulta necesario establecer consensos mínimos que permitan generar protocolos detallados que incluyan el tipo de dato a compartir, la definición de las variables relevadas, la evaluación del error de medición, la forma en que las variables deben ser recolectadas y almacenadas y la información complementaria (o metadatos) que deben acompañarlas, entre otros, a fin de maximizar el potencial de las bases de 
datos y optimizar los recursos invertidos en su obtención.

El interés por establecer protocolos para la obtención de variables antropométricas ha constituido un punto central de la Antropología Biológica. Desde los inicios de las investigaciones de Petrus Camper hacia fines del siglo XVIII, quien definió una de las primeras medidas lineales para describir la variación morfológica humana, el número de variables cuantitativas empleadas se incrementó rápidamente. Como consecuencia, surgió la necesidad de estandarizar las definiciones de las medidas y de las técnicas empleadas en su recolección, de forma tal que fuese posible la comparación de los resultados obtenidos por diversos investigadores. Para ello se crearon comités de especialistas y se llevaron a cabo una serie de reuniones científicas -entre ellas la convención de Frankfurt (1877), la Convención Internacional de Mónaco (1906), la Convención de Génova (1912) y la Convención de Ohio (1967)- (Duckworth 1919; Hertzberg 1968; Hrdlička 1936; Papillault 1919). Asimismo, se reconoció la importancia de establecer el número mínimo de datos que era preciso relevar en distintas clases de estudios, a fin de caracterizar adecuadamente el problema a investigar.

Más recientemente, luego de la sanción de la ley de protección de sepulturas nativas en EEUU (NAGPRA11990) y en el marco de un cambio en el significado de las colecciones de restos humanos, se publicó el Standard for data collection (Buikstra y Ubelaker 1994). Allí se compiló, a partir de procedimientos desarrollados por diferentes autores, un conjunto mínimo y significativo de datos a relevar antes de ejecutar la restitución de los restos a las comunidades de pertenencia. El mismo incluye definiciones para la obtención de variables métricas lineales, variables no-métricas, patologías, rasgos tafonómicos y modificaciones culturales. Este manual se ha convertido en una referencia obligada que trasciende las fronteras de EEUU ya que se considera que el registro estandarizado de datos potencia su utilidad al hacer comparable la información relevada por diferentes investigadores.

Aunque las ventajas de contar con un número de variables comunes, relevadas por todos los investigadores, son claras en términos de la comparabilidad de los resultados, resulta difícil que el mismo conjunto de datos sea adecuado para abordar problemáticas tan diversas como los estudios ontogenéticos, evolutivos y funcionales. En este sentido, Washburn (1953) ha señalado que a diferencia de la antropología física tradicional que empleaba un único conjunto de medidas para resolver problemas diversos, en el marco de la Nueva Antropología Física cada problema requiere el empleo de variables específicas. El empleo de protocolos de trabajo comunes que simultáneamente incluyan conjuntos flexibles de medidas, de modo tal que resulten adecuados para la resolución de preguntas de investigación particulares, es aún problemático.
La complejidad de este problema se ha incrementado hacia la década de 1990, con el desarrollo de las técnicas de la morfometría geométrica (Adams et al. 2004; Bookstein 1991). Estas técnicas se basan en la obtención de coordenadas cartesianas de puntos anatómicos a partir de imágenes digitales en 2 y 3D. A diferencia del consenso relativo al que se había llegado en el empleo de las medidas lineales, el registro de estas nuevas variables no siguió lineamientos compartidos. Esto ha resultado en una gran disparidad en términos de calidad y cantidad de la información contenida en distintos conjuntos de datos.

Otro aspecto que debe considerarse en el contexto de políticas de acceso abierto es la modalidad de publicación de los datos en los repositorios, es decir, qué clase de datos y en qué formato se publican. A lo largo de más de 100 años de estudios en el campo de la antropología se ha acumulado una gran cantidad de datos de poblaciones humanas actuales, homínidos fósiles y primates. Previamente al desarrollo de soportes en formato digital, la única alternativa para la publicación de los resultados era a través de la impresión de libros y publicaciones periódicas, editados por instituciones educativas, asociaciones profesionales o editoriales privadas. Dado que los trabajos generalmente se basaban en un conjunto reducido de datos, la inclusión de las variables en tablas y anexos era una práctica frecuente, resultando en la disponibilidad de una considerable cantidad de datos sobre medidas craneales y postcraneales de poblaciones actuales y prehistóricas, así como de homínidos fósiles (Roberts 1953; Wolpoff 1971). Si bien estos datos se encuentran potencialmente disponibles, su acceso está limitado a la obtención de la publicación original y a la transformación de los mismos a un formato adecuado para su análisis estadístico, lo que resulta una tarea ardua y sujeta a diversas fuentes de error.

La tecnología digital ha facilitado el almacenamiento y transferencia de datos, sin embargo, hasta el presente muy pocas bases de datos morfométricos se encuentran disponibles en repositorios on line. En este sentido, se destaca el trabajo pionero de William W. Howells quien en 1996 publicó on line una base de datos de medidas lineales craneofaciales, con información sobre más de 2000 individuos procedentes de 28 poblaciones de todo el mundo (http://konig.la.utk.edu/howells.htm; Howells 1996). Más recientemente se han puesto a disposición bases de medidas lineales del esqueleto postcraneal de poblaciones aborígenes del Nuevo y Viejo Mundo (http:// web.utk.edu/ auerbach/GOLD.htm; Auerbach y Ruff 2004) y datos dentales, craneales y postcraneales de poblaciones modernas y prehistóricas de Australia, China y Europa (http://www-personal.une.edu.au/ pbrown3/ resource.html). Además de estos esfuerzos individuales, se observa un creciente interés por crear bases de datos primarios en el marco de consorcios internacionales conformados alrededor de problemáticas particulares. Por ejemplo, la base de medidas lineales craneales, dentales y postrcaneales de Cercopithecidos desarrollada por el New 
York Consortium in Evolutionary Primatology (PRIMO, PRImate Morphology Online database; http://primo. nycep.org; Delson et al. 2007), la base de datos sobre marcadores de estilo y calidad de vida biológica (Global History of Health Project, dirigido por los Drs. Steckel, Larsen, Sciuli y Walker; http://global.sbs.ohio-state. edu/), la base de datos craneofaciales correspondiente al FaceBase consortium (Hochheiser et al. 2011), entre otras.

Por su parte, el creciente acceso a nuevas tecnologías para la captura de estructuras morfológicas, como la fotografía digital, tomografía computada, resonancia magnética y escáner láser, ha abierto la posibilidad de hacer públicas copias de alta calidad de los materiales estudiados que pueden ser compartidas en diversos formatos digitales como DICOM, ply, etc. (Bruner 2009; Hallgrímsson et al. 2008; Kullmer 2008; Sumner y Riddle 2009). Estos métodos no invasivos posibilitan la creación de repositorios de las muestras, que de otra forma serían difícilmente accesibles, a partir de las cuales cada investigador puede relevar el conjunto de variables más adecuado para su problema de investigación. La constitución de estas muestras "virtuales" presenta diversas ventajas desde el punto de vista de las instituciones que albergan las colecciones ya que previene la manipulación constante de los materiales y permite preservar una copia altamente fiable en caso de pérdida, destrucción, repatriación, restitución, etc. Por lo tanto, las nuevas técnicas de análisis de imágenes no sólo posibilitan la observación, descripción y cuantificación de nuevos rasgos, sino que al mismo tiempo ofrecen nuevas perspectivas para la conservación de las colecciones (Gilissen 2009). Como ejemplo de estos nuevos repositorios virtuales pueden citarse la base digital de cráneos primates de la Universidad de Tokio (http:// www.pri.kyoto-u.ac.jp/dmm/WebGallery/index.html) y la colección virtual de referencia de vertebrados creada por miembros del Proyecto de Zooarqueología Virtual del Ártico (VZAP por su sigla en inglés, http://vzap.iri.isu.edu/ ViewPage. aspx?id=230).

En líneas generales, se observa un gran avance en el data sharing de variables morfométricas en antropología, sin embargo, diversos aspectos restan ser resueltos. En primer lugar, aun no se ha alcanzado un consenso general acerca del tipo de datos a archivar y el formato en que los mismos deberían ser presentados. El rápido avance de la informática plantea el desafío de guardar los datos en formatos y soportes que puedan ser utilizados en el futuro, ya que los formatos de los archivos se desactualizan rápidamente (una alternativa es emplear formatos simples como txt, en lugar de hojas de cálculo o bases de datos complejas). Es importante avanzar en este sentido para arribar a criterios comunes. Asimismo, el almacenaje de grandes volúmenes de datos plantea la necesidad de contar con repositorios que permitan realizar búsquedas, cruzar información etc. Si los datos se publican únicamente en páginas personales o como datos suplementarios de las publicaciones se produce una gran dispersión de la información y por lo tanto, se dificulta el empleo conjunto de múltiples bases. La conformación de bases con tales características requiere de una infraestructura adecuada, la incorporación en los programas de financiamiento de los fondos requeridos para generar y mantener dichas bases, el trabajo conjunto con especialistas en el manejo de datos y la articulación entre los editores de las publicaciones científicas, asociaciones profesionales e instituciones públicas, como universidades, bibliotecas, etc (Torres-Salinas et al. 2012). En este sentido, el desarrollo de plataformas on line, similares a las que posibilitan actualmente el mantenimiento de revistas científicas open access (e.g. Portal de revistas de la UNLP, Portal de publicaciones científicas y técnicas del CAICYT) con un bajo costo de mantenimiento, puede constituir una alternativa viable para la generación de repositorios de datos científicos.

\section{Estado actual en Argentina y agenda}

En el seno de la antropología biológica argentina aún no se ha instalado la discusión sobre el data sharing. En este sentido, a pesar del incremento registrado en los últimos años en la cantidad de subsidios otorgados por los organismos nacionales de financiamiento y el concomitante aumento en la generación de datos, aún no se han delineado políticas respecto al manejo de los mismos. Por otro lado, las revistas científicas y las instituciones locales tampoco propician la publicación libre de las bases de datos que sustentan los trabajos, ni de la información generada a partir del estudio de las colecciones bajo su custodia.

Algunas gestiones con el fin de avanzar hacia la creación de un repositorio de datos bioantropológicos fueron iniciadas durante el año 2010 por la Asociación de Antropología Biológica Argentina ante el Ministerio de Ciencia, Tecnología e Innovación Productiva de la Nación Argentina para incluir estos datos dentro del Sistema Nacional de Datos Biológicos (http://sistemasnacionales. mincyt.gob.ar/). Este Sistema tiene por objetivos promover el intercambio de información a través de una red nacional de datos, acordar políticas sobre la calidad de los mismos, incrementar y mejorar la accesibilidad de la información manteniéndola actualizada, dotar de proyección internacional a los datos a través de redes virtuales. Por su parte, las instituciones participantes se comprometen, entre otras cosas, a asegurar la conservación de las colecciones y del contenido y la calidad de los datos. A pesar de lo promisorio de esta iniciativa, aún se ha avanzado muy poco en este sentido.

Esta situación puede atribuirse a múltiples factores vinculados principalmente a la disponibilidad de recursos, a las prácticas de los investigadores y a la ausencia de un marco ético-legal que regule la creación y uso de los repositorios de datos.

Recientemente, uno de los organismos más importante 
de financiamiento de la investigación científica en Argentina (CONICET) realizó una encuesta entre los investigadores del área de las ciencias sociales $-43 \%$ correspondiente a las áreas de Historia, Geografía y Antropología- para obtener información sobre las fuentes que utilizan y su nivel de digitalización. Los resultados indicaron que un alto porcentaje de los investigadores $(36,3 \%)$ no ha digitalizado sus datos por falta de infraestructura, herramientas (hardware/software) y/o personal entrenado. Asimismo, el $80 \%$ señaló que no existen archivos institucionales en el lugar de trabajo y por lo tanto, la información se encuentra generalmente dispersa en archivos personales, dificultando su amplia difusión y acceso.

Por otro lado, temas como el reconocimiento a las instituciones y organismos que sustentan la obtención de datos y/o la digitalización de los materiales, a las instituciones que albergan los mismos, así como a los investigadores involucrados, permanecen aún librados a acuerdos particulares ${ }^{1}$. Esto conduce a que los investigadores se muestren renuentes a compartir los datos que generan. En el caso especial de materiales considerados sensibles, como los restos humanos ${ }^{2} \mathrm{y}$ particularmente aquellos correspondientes a pueblos originarios, debe tenerse en cuenta además el derecho de que las comunidades de pertenencia a intervenir en todo proceso que involucre el estudio de los restos de sus antepasados (Código ICOM 2007; Ley 25.517, art.3). Este derecho está contemplado en la Constitución Nacional art. 75, inciso 17, en leyes provinciales sobre el patrimonio cultural y la ley Nacional 25.743, y ha sido reconocido en declaraciones de sociedades científicas y en el "Código deontológico para el estudio, conservación y gestión de restos humanos de poblaciones del pasado" de la Asociación de Antropología Biológica Argentina. Sin embargo, aún no se han establecido los procedimientos a seguir para su efectiva implementación.

Una mención especial merecen los datos antropométricos relevados en poblaciones actuales. En los últimos años se han desarrollado en nuestro país numerosos proyectos tendientes a evaluar el estado nutricional de la población infanto-juvenil, a través del relevamiento de variables antropométricas como talla, peso, etc. (Bolzan et al. 2005; Oyhenart et al. 2008). El libre acceso a estos datos resulta de gran importancia, no sólo para la comunidad científica, sino también para los órganos gubernamentales que diseñan políticas de desarrollo social, y tiene además implicancias prácticas para el diseño ergonómico de mobiliario, indumentaria, prótesis, etc. Este ejemplo remarca el potencial que la publicación de las bases de datos tiene para contribuir al desarrollo científico, social y económico de la sociedad.

\footnotetext{
${ }^{1}$ En este sentido, el sistema Creative Commons, u otro similar, podría contribuir a regular los términos y condiciones de uso de los datos digitales.

2 Definición de material sensible del Código de Deontología del ICOM para Museos (2006)
}

Sobre la base de la revisión realizada aquí, surgen una serie de cuestiones que requieren aún un mayor debate y que constituyen la agenda de trabajo futura a fin de extender la práctica de libre acceso a los datos morfométricos generados en el campo de la antropología biológica. Particularmente, consideramos que es necesario profundizar este debate a fin de: -lograr un consenso respecto a la definición y especificación de los tipos de datos a recolectar y la forma de almacenarlos; -generar protocolos de obtención de datos que incluyan estandarización de las definiciones e instrumentos a utilizar; - promover acciones tendientes a contar con infraestructura (hardware y software) y personal capacitado en la curación de datos; -alcanzar un mayor consenso acerca de los aspectos ético-legales; -diseñar e implementar políticas que propicien la implementación de redes colaborativas.

La ejecución de una agenda de trabajo orientada a alcanzar el objetivo de un genuino data sharing contribuirá a potenciar las capacidades científicas y a expandir la transferencia de conocimientos generados por la antropología biológica al conjunto de la sociedad. Este proceso plantea importantes desafios y consituirá un foco central de debate en el futuro próximo de la disciplina.

\section{Organismos de financiamiento}

CONICET; CIHR Training Program in Genetics, Child Development (Health Alberta Children's Hospital Research Institute for Child and Maternal Health); Alberta Innovates Health Solutions; American Association of Physical Anthropology.

\section{Bibliografía}

Adams, D.C., F.J. Rohlf, D.E. Slice. 2004. Geometric morphometrics: ten years of progress following the 'revolution'. Italian Journal of Zoology, 71:5-16.

Anton, S.C., C.B. Ruff. 2011. Data archiving as a prerequisite for publication. American Journal Physical Anthropology, 144 Sup 52: 77.

Arzberger, P., P. Schroeder, A. Beaulieu, G. Bowker, K. Casey, L. Laaksonen, D. Moorman, P. Uhlir y P. Wouters. 2004. Promoting Access to Public Research Data for Scientific, Economic, and Social Development. Data Science Journal, 3:135-152

Auerbach, B.M. y C.B. Ruff. 2004. Human body mass estimation: A comparison of "morphometric" and "mechanical" methods. American Journal Physical Anthropology, 125:331-342.

Bookstein, F. L. 1991. Morphometric tools for landmark data: geometry and biology. Cambridge University Press, New York.

Bruner, E. 2009. New tools and old perspectives in the management of the fossil resources. Journal of Anthropological Sciences, 87: 227-228.

Buikstra, J.E., D. Ubelaker. 1994. Standards for Data Collection from Human Skeletal Remains. Arkansas Archeological Survey 
Research Series. 44 Archeological Survey, Fayetteville. Arkansas.

Delson, E., W.E.H. Harcourt-Smith, S.R. Frost y C.A. Norris. 2007. Databases, data access and data sharing in palaeoanthropology: first steps. Evolutionary Anthropology, 16:161-163.

Duckworth, W.L.H. 1919. The international agreement for the unification of anthropometric measurements to be made on the living subject. American Journal Physical Anthropology, 2:61-67.

Fairbairn, D.J. 2010. The advent of mandatory data archiving. Evolution, 65-1:1-2.

Gilissen, E. 2009. Museum collections, scanning, and data access. Journal of Anthropologicl Science, 87:223-226.

Hallgrímsson, B., M.L. Zelditch, T. Parsons, E. Kristensen, N.M. Young y S.K. Boyd. 2008. Morphometrics and biological anthropology in the postgenomic age. Katzenberg, M.A. y S.R. Saunders, editores, Biological anthropology of the human skeleton, 207-235, John Wiley \& Sons, New Jersey.

Hertzberg, H.T.E. 1968. The conference on standardization of anthropometric techniques and terminology. American Journal Physical Anthropology, 28:1-16.

Hochheiser, H., B.J.Aronow, K. Artinger, T.H. Beaty, J.F. Brinkley, Y. Chai., D. Clouthier, M.L. Cunningham, M. Dixon, L.R. Donahue, et al. 2011. The FaceBase Consortium: a comprehensive program to facilitate craniofacial research. Developmental Biolology, 355: 175-182.

Howells, W.W. 1996. Howells' Craniometric Data on the Internet. American Journal Physical Anthropology, 101:441-442.

Hrdlička, A. 1936. Anthropometry. The anthropometric committee of the American Association of Physical Anthropologists. American Journal Physical Anthropology, 21:287-300.

ICOM, 2006. Código de deontología del ICOM para los museos. Aprobado en la $15^{a}$ Asamblea General del ICOM, en Buenos Aires 1986. Modificado en la $20^{\mathrm{a}}$ y $21^{\mathrm{a}}$ Asambleas Generales 2001 en Barcelona y 2004 en Seúl.

Kullmer, O. 2008. Benefits and risks in virtual anthropology. Journal of Anthropological Science, 86:205-207.

Lossau, N. 2008. The Concept of Open Access. En "Open Access - Opportunities and challenges - A handbook", European Commission and the German Commission for UNESCO, traducido de la versión original en alemán publicada por la comisión alemana de UNESCO.

Moore, A.J., M.A. Mcpeek, M.D. Rausher, L. Riesenberg, y M.C.Whitlock. 2010. The need for archiving data in evolutionary biology. Journal Evolutionary Biology, 23:659-660.
OECD. 2007. Principles and guidelines for access to research data from public funding. http://www.oecd.org/ dataoecd/9/61/38500813.pdf

Oyhenart, E.E., Dahinten, S., Alba, J.A., Alfaro, E.L., Bejarano, I.F., Cabrera, G.E., Cesani, M.F., Dipierri, J.E., Forte, L.M., Lomaglio, D.B., et al. 2010. Estado nutricional infanto juvenil en seis provincias de Argentina: variación regional. Revista Argentina de Antropología Biológica, 10: 5-62.

Papillault, G. 1919. The international agreement for the unification of craniometric and cephalometric measurements. American Journal Physical Anthropology, 2:46-60.

Rausher, M.D., M.A. McPeek, A.J. Moore, L. Rieseberg, y M.C. Whitlock. 2010. Data archiving. Evolution 64:603-604.

Rieseberg, L., T. Vines, y N. Kane. 2010. Editorial and retrospective. Molecular Ecolology, 19:1-22

Roberts, D.F. 1953. Body weight, race and climate. American Journal Physical Anthropology, 11:533-558.

Rodrigo, A., Alberts, S., Cranston, K., y Kingsolver, J. 2013. Science incubators: synthesis centers and their role in the research ecosystem. PloS biology, 11(1), 1-3. doi:10.1371/ journal.pbio. 1001468

Sumner, T.A., A.T.R. Riddle. 2009. Remote anthropology: Reconciling research priorities with digital data sharing. Journal of Anthropological Science, 89:1-3.

Uhlir, P.F., P. Schröder. 2007. Open data for global science. Data Science Journal 6:35-53.

Torres-Salinas, D. 2010 Compartir datos (data sharing) en ciencia: contexto de una oportunidad. Anuario Thinkepi, 262265.

Torres-Salinas, D., N. Robinson-García y A. Cabezas-Clavijo. 2012. Compartir los datos de investigación en ciencia: introducción al data sharing. El profesional de la información, v. 21, n. 2.

UNESCO. 2008. Open access: opportunities and challenges - A handbook. European Commission and the German Commission for UNESCO. http://www.unesco.de/openaccess-en.html.

Washburn, S. 1953. The strategy of the Physical Anthropology. Kroeber, A., Anthropology Today, editor, 714-727. Chicago University Press, Chicago.

Whitlock, M.C. 2011. Data archiving in ecology and evolution: best practices. Trends in Ecolology and Evolution, 26:61-65

Wolpoff, M.H. 1971. Metric trends in hominid dental evolution. Case Western Reserve Studies in Anthropology 2. Case Western Reserve University Press, Cleveland. 